\title{
A Class of Hessenberg Matrices with Known Pseudoinverse and Drazin Inverse
}

\author{
By Inderjit Singh, George Poole and Thomas Boullion
}

\begin{abstract}
In this paper, a class of Hessenberg matrices is presented for adoption as test matrices. The Moore-Penrose inverse and the Drazin inverse for each member of this class are determined explicitly.
\end{abstract}

1. Introduction. Most numerical problems associated with solving a system of linear equations involve only rational numbers. However, square matrices over the real number field are considered in this paper.

Howell and Gregory [6] have shown how to avoid problems which arise in solving the matrix equation $A x=b$ as a result of rounding errors in computer schemes. Specifically, they have shown how to use residue arithmetic to avoid ill-conditioned problems. Using a similar approach, Stallings and Boullion [12] have shown how to significantly reduce rounding errors in computer schemes which compute the Moore-Penrose inverse (pseudoinverse) for a given matrix. However, the rounding errors are not necessarily completely eliminated.

Chow [2] has presented a class of Hessenberg matrices which may be used as test matrices in checking the accuracy of matrix inversion programs. In this paper, a class of Hessenberg matrices is presented such that the pseudoinverse and Drazin inverse can be explicitly computed for each member. Furthermore, the eigenvalues and eigenvectors are known for the members of this class. Therefore, it appears reasonable that such a class of matrices may be useful as test matrices.

2. Definitions and Notation. One should distinguish between the class of matrices in [2] which are offered as test matrices and the class given below. Only square matrices over the real number field are considered.

Definition 2.1. The pseudoinverse of a matrix $A$ is the unique solution $A^{+}$of the four matrix equations $A X A=A, X A X=X,(A X)^{T}=A X$ and $(X A)^{T}=X A$ where ( ) ${ }^{T}$ denotes the matrix transpose.

Definition 2.2. The index of a matrix $A$ is the smallest nonnegative integer Ind $(A)=k$ such that $\operatorname{rank}\left(A^{k}\right)=\operatorname{rank}\left(A^{k+1}\right)$.

Definition 2.3. The Drazin inverse of a matrix $A$ is the unique solution $A^{D}$ of the three matrix equations $A X=X A, X A X=X, A^{k+1} X=A^{k}$, where $\operatorname{Ind}(A)=k$. 
$H_{n}$ shall denote the Hessenberg matrix of order $n$, where

$$
H_{n}=\left[\begin{array}{ccccc}
\alpha & 1 & \cdot & \cdot & \cdot \\
\alpha^{2} & \alpha & 1 & \cdot & \cdot \\
\alpha^{3} & \alpha^{2} & \alpha & \cdot & \cdot \\
\cdot & \cdot & \cdot & \cdot & \cdot \\
\cdot & \cdot & \cdot & \cdot & 1 \\
\alpha^{n} & \alpha^{n-1} & \cdot & \cdot & \alpha
\end{array}\right],
$$

and $\alpha$ is an arbitrary real number.

The eigenvalues of $H_{n}$ were determined in [2]. $H_{n}$ has $k=[n / 2]$ eigenvalues equal to 0 (where $[n / 2]$ denotes the largest integer not exceeding $n / 2$ ) and whose remaining eigenvalues are

$$
4 \alpha \cos ^{2}\left(\frac{m \pi}{n+2}\right), \quad m=1,2, \ldots, n-k .
$$

The reader can refer to [4] for the corresponding eigenvectors.

3. Pseudoinverse of $H_{n}$. There are several algorithms available for computing $\left(H_{n}\right)^{+},[1],[3],[5],[10]$. The general form for $\left(H_{n}\right)^{+}$is presented in this section. Case $1(n=2)$. If $H_{2}$ is the matrix

$$
H_{2}=\left[\begin{array}{cc}
\alpha & 1 \\
\alpha^{2} & \alpha
\end{array}\right], \text { then }\left(H_{2}\right)^{+}=\left[\begin{array}{cc}
\frac{\alpha}{\left(\alpha^{2}+1\right)^{2}} & \frac{\alpha^{2}}{\left(\alpha^{2}+1\right)^{2}} \\
\frac{1}{\left(\alpha^{2}+1\right)^{2}} & \frac{\alpha}{\left(\alpha^{2}+1\right)^{2}}
\end{array}\right] .
$$

This can be easily verified by direct substitution into the four defining equations. Case $2(n \geqslant 3)$. If $H_{n}$ is the Hessenberg matrix of order $n \geqslant 3$, then

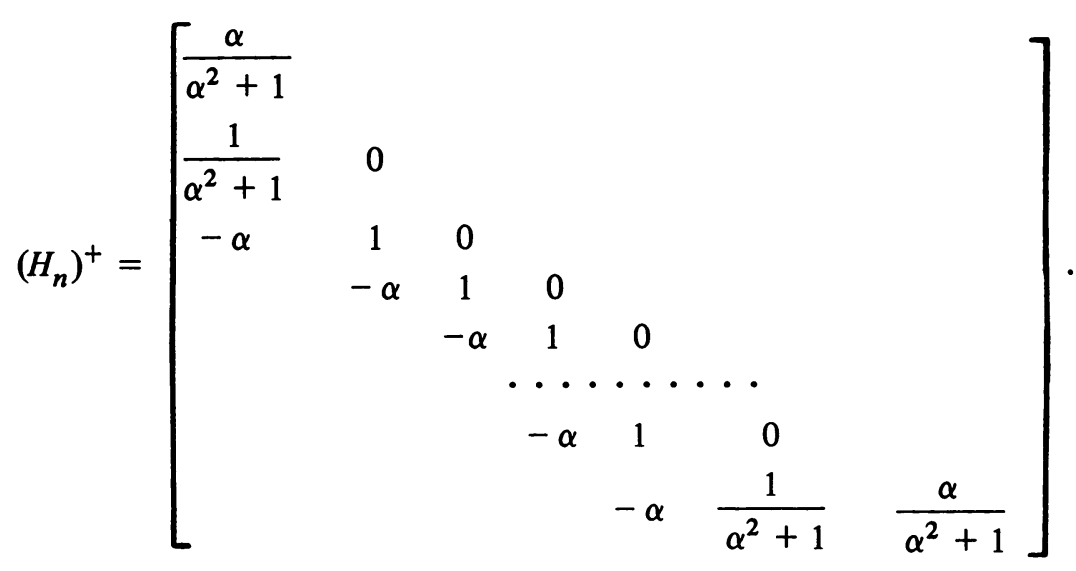

As in Case 1 , this can be easily verified by direct substitution after noting 


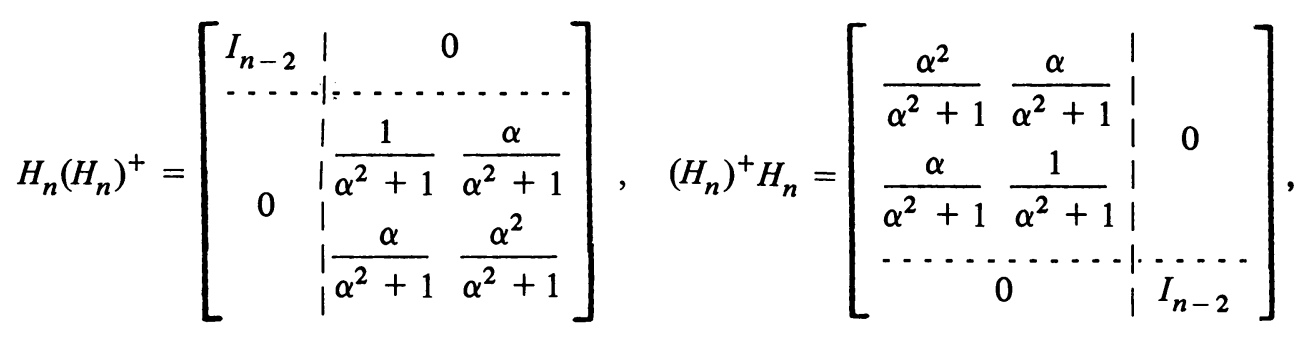

where $I_{n-2}$ is the identity matrix of order $n-2$.

4. Drazin Inverse of $H_{n}$. The index of $H_{n}$ is first determined. If $\alpha=0$, then $H_{n}$ is nilpotent and Ind $\left(H_{n}\right)=n$.

Proposition. If $\alpha \neq 0$, then Ind $\left(H_{n}\right)=[n / 2]$.

Proof. Meyer [7] has shown that $k$ is the index of $H_{n}$ if $k$ is the smallest integer such that limit $\epsilon_{\epsilon \rightarrow 0} \epsilon^{k}\left(H_{n}+\epsilon I_{n}\right)^{-1}$ exists. From [2] it is known that, if $\left(h_{i j}\right)=$ $\left(H_{n}+\epsilon I_{n}\right)^{-1}$, then

and

$$
h_{i j}=\frac{(-1)^{i+j} \Delta_{i-1}^{\prime} \Delta_{n-j+1}}{\epsilon \Delta_{n}^{\prime}}, \text { if } i \leqslant j
$$

where

$$
h_{i j}=\frac{-\alpha(\alpha \epsilon)^{i-j} \Delta_{j-2}^{\prime} \Delta_{n-i}}{\Delta_{n}^{\prime}}, \text { if } i>j
$$

$$
\begin{gathered}
\Delta_{0}=1, \quad \Delta_{0}^{\prime}=1, \quad \Delta_{-1}^{\prime}=1 / \epsilon \\
\Delta_{1}=\epsilon, \quad \Delta_{1}^{\prime}=\alpha+\epsilon \\
\Delta_{t}=\epsilon \Delta_{t-1}+\alpha \epsilon \Delta_{t-2}, \quad \Delta_{t}^{\prime}=\Delta_{t}+\alpha \Delta_{t-1} .
\end{gathered}
$$

Each $\Delta_{i}$ is a polynomial of degree $i$ in $\epsilon$.

First, in computing the

$$
\operatorname{limit}_{\epsilon \rightarrow 0} \epsilon^{k} h_{i j}=\operatorname{limit}_{\epsilon \rightarrow 0} \frac{\epsilon^{k}(-1)^{i+j} \Delta_{i-1}^{\prime} \Delta_{n-j+1}}{\epsilon \Delta_{n}^{\prime}}
$$

(when $i \leqslant j$ ) attention is directed to the terms of smallest power in $\epsilon$ of $\Delta_{t}$ and $\Delta_{t}^{\prime}$. Observe that

(1) The exponent of $\epsilon$ in the term of smallest degree in $\Delta_{t}$ is $t / 2$ when $t$ is even and $(t+1) / 2$ otherwise,

(2) the exponent of $\epsilon$ in the term of smallest degree in $\Delta_{t}^{\prime}$ is $[t / 2]$.

Since, for a given $n, \epsilon \Delta_{n}^{\prime}$ is fixed, the value of $k$ depends on $\Delta_{i-1}^{\prime} \Delta_{n-j+1}$. In this polynomial, the exponent of $\epsilon$ is minimum when $i$ is smallest and $j$ is largest. Therefore, the integer $k$ for which limit $\epsilon_{\epsilon \rightarrow 0} \epsilon^{k} h_{1 n}$ exists is also an integer for which limit $_{\epsilon \rightarrow 0} \epsilon^{k} h_{i j}$ exists $(i \leqslant j)$. Now

$$
\operatorname{limit}_{\epsilon \rightarrow 0} \epsilon^{k} h_{1 n}=\operatorname{limit}_{\epsilon \rightarrow 0} \frac{\epsilon^{k}(-1)^{1+n} \Delta_{0}^{\prime} \Delta_{1}}{\epsilon \Delta_{n}^{\prime}}=\operatorname{limit}_{\epsilon \rightarrow 0} \frac{(-1)^{1+n} \epsilon^{k+1}}{\epsilon \Delta_{n}^{\prime}}
$$

exists if $k \geqslant[n / 2]$. 
Second, when $i>j$, a similar argument will show that limit $\epsilon_{\rightarrow 0} \epsilon^{k} h_{i j}$ exists whenever limit $\epsilon_{\epsilon \rightarrow 0} \epsilon^{k} h_{n 1}$ exists, which is true when $k \geqslant[n / 2]$.

Therefore, limit $\epsilon_{\epsilon \rightarrow 0} \epsilon^{k}\left(H_{n}+\epsilon I_{n}\right)^{-1}$ exists whenever $k \geqslant[n / 2]$. To see that $k$ is the smallest such integer observe that

$$
\operatorname{limit}_{\epsilon \rightarrow 0} \epsilon^{[n / 2]-1} h_{1 n}=\operatorname{limit}_{\epsilon \rightarrow 0} \frac{\epsilon^{[n / 2]-1}(-1)^{1+n} \epsilon}{\epsilon \Delta_{n}^{\prime}}
$$

does not exist since the exponent of $\epsilon$ in the term of smallest degree in $\Delta_{n}^{\prime}$ is $[n / 2]$. This completes the proof.

The Drazin inverse is now determined for $H_{n}$, using elementary divisor theory (see [8]) and a technique described in [9]. Consider the characteristic matrix $\left(H_{n}-\lambda_{n}\right)$. Let $P_{n}(\lambda)$ denote the characteristic polynomial of $H_{n}$ where $P_{n}(\lambda)=$ $\operatorname{det}\left(H_{n}-\lambda_{n}\right)$. Also,

$$
P_{n}(\lambda)=(\alpha-\lambda) P_{n-1}(\lambda)+\sum_{j=1}^{n-1}(-1)^{n-j} \alpha^{n+1-j} P_{j-1}(\lambda)
$$

where $P_{0}(\lambda)=1, P_{1}(\lambda)=\alpha-\lambda$, and $P_{n}(\lambda)+\lambda P_{n-1}(\lambda)+\alpha \lambda P_{n-2}(\lambda)=0$ (expanding by rows). The solution of this last equation [4] is

$$
P_{n}(\lambda)=(-\alpha)^{n} 2^{n-1}(\lambda / 4 \alpha)^{(n-1) / 2} \frac{\sin \left[(n+2) \cos ^{-1}(\lambda / 4 \alpha)^{1 / 2}\right]}{\sin \left[\cos ^{-1}(\lambda / 4 \alpha)^{i / 2}\right]}
$$

Since all determinantal divisors $d_{i}$ corresponding to $H_{n}$ are equal to one except $d_{n}=$ $\operatorname{det}\left(H_{n}-\lambda_{n}\right)$, the minimum polynomial of $H_{n}$ is $P_{n}(\lambda)$. Suppose

$$
H_{n}=p^{-1}\left[\begin{array}{ll}
B & 0 \\
0 & N
\end{array}\right] P
$$

where $B$ is nonsingular and $N$ is nilpotent. Since $\operatorname{Ind}\left(H_{n}\right)=[n / 2]$, the minimum polynomial of $B$ is $f(\lambda)=P_{n}(\lambda) / \lambda^{[n / 2]}$ or

$$
f(\lambda)= \begin{cases}-\alpha^{(n+1) / 2} \frac{\sin \left[(n+2) \cos ^{-1}(\lambda / 4 \alpha)^{1 / 2}\right]}{\sin \left[\cos ^{-1}(\lambda / 4 \alpha)^{1 / 2}\right]}, & \text { if } n \text { is odd, } \\ \alpha^{(n+1) / 2} \frac{\lambda^{-1 / 2} \sin \left[(n+2) \cos ^{-1}(\lambda / 4 \alpha)^{1 / 2}\right]}{\sin \left[\cos ^{-1}(\lambda / 4 \alpha)^{1 / 2}\right]}, & \text { if } n \text { is even. }\end{cases}
$$

The degree of $f(\lambda)$ is $(n+1) / 2$ or $n / 2$ depending on whether $n$ is odd or even. If $f(\lambda)=a_{t} \lambda^{t}+a_{t-1} \lambda^{t-1}+\cdots+a_{1} \lambda+a_{0}=0$, then set

$$
g(\lambda)=\lambda^{-1}=-\frac{1}{a_{0}}\left(a_{t} \lambda^{t-1}+\cdots+a_{2} \lambda+a_{1}\right) .
$$

Therefore [9], if $h(\lambda)=\lambda^{[n / 2]} g^{[n / 2]+1}(\lambda)$, then $\left(H_{n}\right)^{D}=h\left(H_{n}\right)$. If $\alpha=0$, $\left(H_{n}\right)^{D}=0$.

Example. Consider

$$
H=\left[\begin{array}{lll}
1 & 1 & 0 \\
1 & 1 & 1 \\
1 & 1 & 1
\end{array}\right]
$$


where $\alpha=1$ and $n=3$.

The index of $H$ is 1 , so that

$$
f(\lambda)=-\frac{\sin \left[5 \cos ^{-1}(\lambda / 4)^{1 / 2}\right]}{\sin \left[\cos ^{-1}(\lambda / 4)^{1 / 2}\right]} .
$$

If $\lambda=4 \cos ^{2}(\theta)$, then

$$
\begin{aligned}
f(\lambda) & =-\frac{\sin (5 \theta)}{\sin (\theta)}=-\frac{1}{\sin (\theta)}\left[8 \cos ^{2}(\theta) \sin (\theta)-16 \sin ^{3}(\theta) \cos ^{2}(\theta)-3 \sin (\theta)+4 \sin ^{3}(\theta)\right] \\
& =-\left(8 \cos ^{2}(\theta)-16 \sin ^{2}(\theta) \cos ^{2}(\theta)-3+4 \sin ^{2}(\theta)\right) .
\end{aligned}
$$

Upon substitution of $\cos (\theta)=(\lambda / 4)^{1 / 2}, \sin (\theta)=((4-\lambda) / 4)^{1 / 2}, f(\lambda)=-\left(\lambda^{2}-3 \lambda+1\right)$ is the minimum polynomial for $B$. Therefore, $h(\lambda)=\lambda g^{2}(\lambda)=\lambda(3-\lambda)^{2}=9 \lambda-6 \lambda^{2}$ $+\lambda^{3}$ and

$$
H^{D}=h(H)=9 H-6 H^{2}+H^{3}=\left[\begin{array}{rrr}
2 & 2 & -3 \\
-1 & -1 & 2 \\
-1 & -1 & 2
\end{array}\right]
$$

Mathematics Department

Kansas State Teachers College

Emporia, Kansas 66801

Mathematics Department

Texas Tech University

Lubbock, Texas 79409

1. A. BEN-ISRAEL, "An iterative method for computing the generalized inverse of an arbitrary matrix," Math. Comp., v. 19, 1965, pp. 452-455. MR 31 \#4152.

2. T. S. CHOW, "A class of Hessenberg matrices with known eigenvalues and inverses," SIAM Rev., v. 11, 1969, pp. 391-395. MR 40 \#5627.

3. H. P. DECELL, "An application of the Cayley-Hamilton theorem to generalized matrix inversion," SIAM Rev., v. 7, 1965, pp. 526-528. MR 33 \#2656.

4. G. FAIRWEATHER, "On the eigenvalues and eigenvectors of a class of Hessenberg matrices," SIAM Rev., v. 13, 1971, pp. 220-221. MR 44 \#4016.

5. M. R. HESTENES, "Inversion of matrices by biorthogonalization and related results," J. Soc. Indust. Appl. Math., v. 6, 1958, pp. 51-90. MR 19, 1080.

6. J. A. HOWELL \& R. T. GREGORY, "An algorithm for solving linear algebraic equations using residue arithmetic. I," BIT, v. 9, 1969, pp. 200-224. MR 41 \#6388a.

7. C. D. MEYER, "Limits and the index of a square matrix," SIAM J. Appl. Math., v. 26, 1974, pp. 469-478.

8. M. NEWMAN, Integral Matrices, Academic Press, New York, 1972.

9. G. POOLE \& T. BOULLION, "The Drazin inverse and a spectral inequality of Marcus, Minc and Moyls," J. Optimization Theory Appl. (To appear.)

10. L. D. PYLE, "Generalized inverse computations using the gradient projection method," J. Assoc. Comput. Mach., v. 11, 1964, pp. 422-428. MR 30 \#2670.

11. C. RAO \& S. MITRA, Generalized Inverse of Matrices and Applications, Wiley, New York, 1971.

12. W. STALLINGS \& T. BOULLION, "Computation of pseudoinverse matrices using residue arithmetic," SIAM Rev., v. 14, 1972, pp. 152-163. 